\title{
Monochromatic Conical IR Emission from Decaying KrF Laser Filaments in Xenon as Coherent Stimulated Four-Wave Mixing Process
}

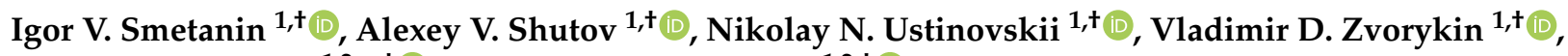 \\ Anna V. Bogatskaya ${ }^{1,2, *,+}$ iD and Alexander M. Popov $1,2,+\mathbb{D}$ \\ 1 P. N. Lebedev Physical Institute, Leninskii Prospect, 53, 119991 Moscow, Russia; \\ smetaniniv@lebedev.ru (I.V.S.); shutovav@lebedev.ru (A.V.S.); ustin5519@yandex.ru (N.N.U.); \\ zvorkinvd@lebedev.ru (V.D.Z.); popov@mics.msu.su (A.M.P.) \\ 2 Physics Department, Michigan State University, 119991 Moscow, Russia \\ * Correspondence: abogatskaya@mics.msu.su \\ + These authors contributed equally to this work.
}

check for updates

Citation: Smetanin, I.V.; Shutov, A.V.; Ustinovskii, N.N.; Zvorykin, V.D.;

Bogatskaya, A.V.; Popov, A.M.

Monochromatic Conical IR Emission from Decaying KrF Laser Filaments in Xenon as Coherent Stimulated Four-Wave Mixing Process. Photonics 2021, 8, 47. https://doi.org/10.3390/ photonics 8020047

Academic Editors: Bonggu Shim and Xiaohui Gao

Received: 24 December 2020

Accepted: 9 February 2021

Published: 12 February 2021

Publisher's Note: MDPI stays neutral with regard to jurisdictional claims in published maps and institutional affiliations.
Abstract: We develop theoretical background for the new nonlinear optical phenomenon of narrowly directed monochromatic IR conical emission which has been recently observed when 248-nm UV filaments propagate in xenon (V. D. Zvorykin, et al., Laser Phys. Lett. 13, 125404 (2016)). We treat it as coherent stimulated four-wave mixing process in which two pump KrF laser photons are converted into the coupled pair of resonance IR $(828 \mathrm{~nm})$ and VUV $(147 \mathrm{~nm})$ photons through $5 p^{5}\left({ }^{2} P_{3 / 2}\right) 6 p[1 / 2]_{0} \rightarrow 5 p^{5}\left({ }^{2} P_{3 / 2}\right) 6 s[3 / 2]_{1}^{\mathrm{o}}$ and $5 p^{5}\left({ }^{2} P_{3 / 2}\right) 6 s[3 / 2]_{1}^{\mathrm{o}} \rightarrow{ }^{1} S_{0}$ transitions. We explore the coherent interaction regime which proceeds at a time scale shorter than transverse relaxation time $T_{2}$. The momentum and energy conservation laws determine the characteristic angle of conical emission. We find that the threshold of this coherent process is determined by the $\mathrm{KrF}$ laser pump pulse area.

Keywords: conical IR emission; coherent four-wave mixing at two-photon absorption; multiple filamentation of sub-picosecond $\mathrm{KrF}$ laser pulse; self-induced defocusing

\section{Introduction}

Conical emission (CE) of frequency-shifted laser radiation is one of the very fascinating effects in nonlinear optics. It was observed for the first time in borosilicate glass [1] and potassium vapor [2] fifty years ago. In most of the cases, CE has been studied in alkali and alkaline-earth vapors with ns-pulse and cw laser excitation when the laser frequency is tuned to the blue side of a resonance transition [3-11]. Femtosecond laserinduced $C E$ has been observed in dense cesium [12] and rubidium [13] vapors. CE with the use of two-photon pumped 3s-3d transition in sodium has been reported in [14]. Various theoretical models have been used to describe the experimental results, which involve a combined effect of four-wave mixing (FWM) and diffractive spreading $[4,11,15,16]$, Cherenkov-type emission [8,17], superfluorescence [18] and self-phase modulation [2,10,12]. Comprehensive study of competition between the resonance-enhanced FWM and amplified spontaneous emission in resonant $C E$ at the $3 d-3 p$ transition in sodium (in which the $3 \mathrm{~s}-3 \mathrm{~d}$ transition is two-photon pumped by the dye laser ns-radiation) have been done by R. W. Boyd and co-authors [19-21]. Numerous works on the two-photon resonanceenhanced FWM and stimulated hyper-Raman scattering in sodium and Xe have been done by W. R. Garrett and co-authors [22-26]. 
CE is a characteristic property of the filamentation phenomenon. Generated in filaments broad-spectrum supercontinuum forms a spatial conical pattern in which each spectral component is emitted at well-defined specific angle $[27,28]$. Modulation instability, $X$-wave and nonlinear focus effects are the dominant mechanisms of filament's CE [29].

Nonlinear interaction of $\mathrm{KrF}$ laser radiation with xenon proceeds in the regime of near-resonant two-photon ${ }^{1} S_{0}-2 p^{5}\left({ }^{2} P_{3 / 2}\right) 6 p[1 / 2]_{0}$ transition which makes it in a definite sense analogous to the mentioned above case of sodium [19-21]. Previously, CE in xenon has been observed as strong coherent continua emissions in the visible and near-infrared $(650-850 \mathrm{~nm})$ and in the UV $(185-400 \mathrm{~nm})$ spectral ranges with a tightly-focused femtosecond $\mathrm{KrF}$ laser radiation at intensities up to $10^{15} \mathrm{~W} / \mathrm{cm}^{2}[30,31]$. It has been found that CE in both these spectral regions appears as a result of non-resonant parametric four-wave difference-frequency conversion process: in the first region, it appears as simultaneous oscillation in the visible and in the VUV (147-155 nm) spectral ranges, whereas in the second region two complementary UV waves are generated. Monochromatic amplified spontaneous IR emission at 828 and $823 \mathrm{~nm}$ has been detected being propagated parallel (and antiparallel) with the pump $\mathrm{KrF}$ laser radiation [30].

We have recently observed, in contrast to the mentioned above experiments [30,31], narrowly directed monochromatic $\mathrm{CE}$ at the $828 \mathrm{~nm}$ wavelength arising from the filaments of $\mathrm{KrF}$ laser radiation at intensities $\sim 10^{11} \mathrm{~W} / \mathrm{cm}^{2}$ [32]. Here, we treat this monochromatic $\mathrm{CE}$ as a resonance-enhanced coherent stimulated four-wave mixing process, in which two pump UV photons are converted into a coupled pair of phase-correlated IR and VUV photons corresponding to the $5 p^{5}\left({ }^{2} P_{3 / 2}\right) 6 p[1 / 2]_{0} \rightarrow 5 p^{5}\left({ }^{2} P_{3 / 2}\right) 6 s[3 / 2]_{1}^{\text {o }}$ and $5 p^{5}\left({ }^{2} P_{3 / 2}\right) 6 s[3 / 2]_{1}^{\text {o }}$ $\rightarrow{ }^{1} S_{0}$ transitions. Unlike conventional FWM CE theories [19-26], we study the coherent interaction regime when the relevant timescale is less than characteristic transverse relaxation time $T_{2}$ in the system. This approach allows us to relate the apex angle of $\mathrm{CE}$ to the momentum and energy conservation laws satisfied in photon conversion process. Being coherent process, CE possesses a threshold which is determined by the pump pulse area.

\section{Generation of Narrowly Directed IR Radiation in the Near-Resonant Interaction of UV Filaments with Xenon}

Experiments are done using the hybrid Ti:Sapphire-KrF laser facility GARPUNMTW [33-35] shown in Figure 1. Frequency-tripled 100 fs laser pulses generated by the Ti:Sapphire laser "Start-248M" (Avesta Project Ltd.) were tuned into B->X transition band of excimer $\mathrm{KrF}$ laser gain medium around $248.4 \mathrm{~nm}$ central wavelength. This short pulses with the energy of $100 \mu \mathrm{J}$ are injected into the two electron-beam pumped $\mathrm{KrF}$ amplifiers separated by a vacuum spatial filter (VSF). After two-pass amplification in the BERDYSH preamplifier and the GARPUN final amplifier, the entire system outputs 248-nm laser pulses with an energy of up to $0.6 \mathrm{~J}$ and a pulse length of $\tau_{p} \approx 1$ ps (FWHM). The final pulse length is stretched due to the group velocity dispersion in the optical elements and air along the amplification path. The laser peak power of $0.6 \mathrm{TW}$ exceeds the critical value for Kerr self-focusing in atmospheric air $(\sim 0.1 \mathrm{GW})$ by 3-4 orders of magnitude resulting in small-scale self-focusing and multiple filamentation. Hundreds of filaments are formed at $15 \mathrm{~m}$ distance from the output window of final amplifier. Visualization of UV radiation is carried out by installing a UV-absorbing K8 glass in the needed position (see Figure 1a). Glass luminescence in the visible range is displayed onto a Spiricon SP620U Beam Profiler (Ophir Photonics) [32]. 


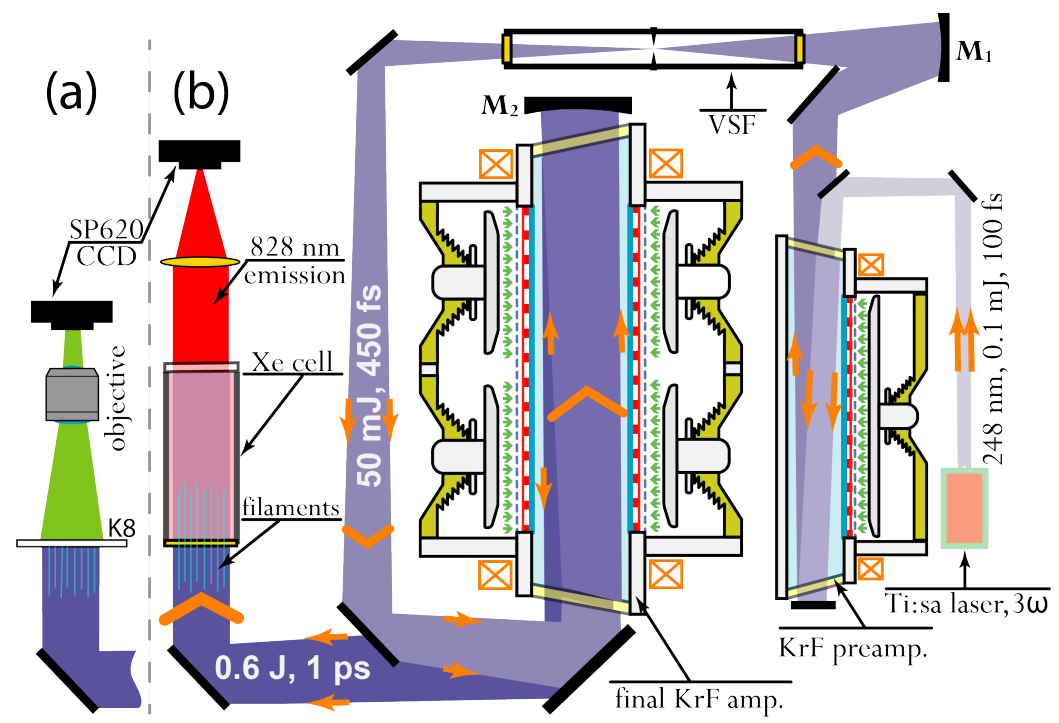

Figure 1. Layout of the experimental setup and scheme for visualization of (a) UV laser beam and (b) IR conical emission.

A filamented UV beam is introduced into a $2.5 \mathrm{~m}$-long gas cell filled with Xe at a pressure varied in the range $0.1-2 \mathrm{~atm}$ (Figure $1 \mathrm{~b}$ ). A telescope formed by mirrors M1 and M2 matches the cross section of the output beam $\sim 12 \times 16 \mathrm{~cm}^{2}$ with the cell input window of $10 \mathrm{~cm}$ in diameter, thus experiments are done with slightly-converging beam. The $\mathrm{CaF}_{2}$ entrance window of the cell is transparent for UV radiation while the exit window of K8 glass serves as UV beam dump. The cell is situated $20 \mathrm{~m}$ away from the final amplifier, such that filaments are already well developed at $5 \mathrm{~m}$ distance before the cell. Luminescence image of UV filaments entering the cell is shown in Figure 2a. The filaments have a diameter $d_{f}=240 \div 340 \mu \mathrm{m}(\mathrm{FWHM})$ and an average peak intensity $I_{f}=(2.0 \pm 0.6) \times 10^{11} \mathrm{~W} / \mathrm{cm}^{2}$, they are surrounded by low-intensity $10^{9} \mathrm{~W} / \mathrm{cm}^{2}$ background radiation. Due to Stimulated Rotational Raman Scattering (SRRS) the UV radiation spectrum is significantly broadened at the end of 20-m air path and covers the range of 247.5-249.8 nm [33,35].
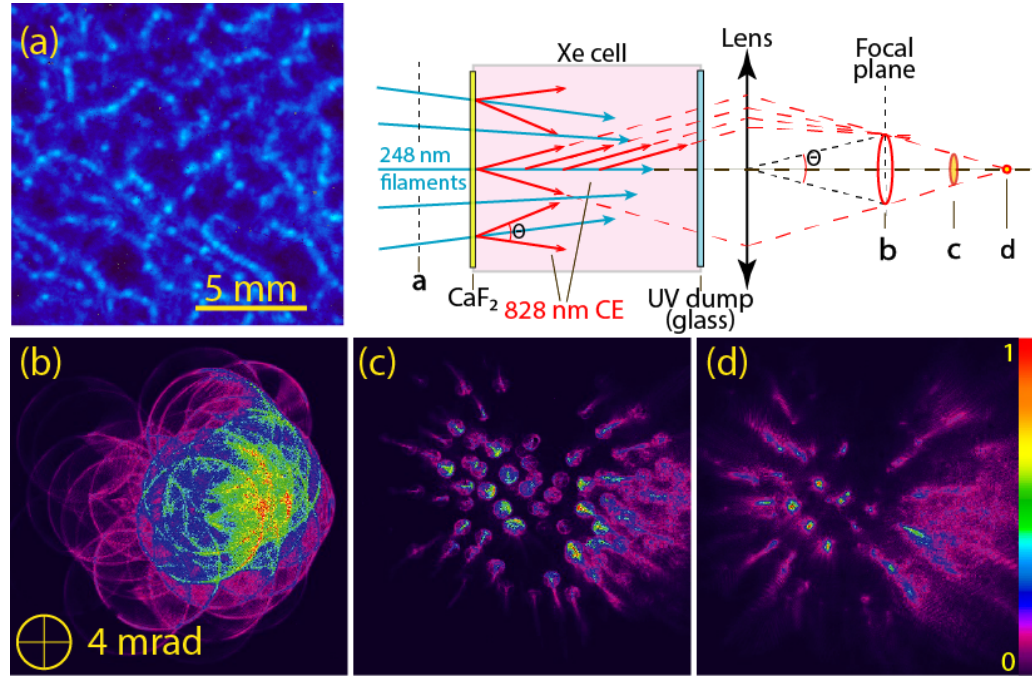

Figure 2. Layout of optical measurements along with the luminescence images of (a) UV filaments entering the cell; (b-d) IR CE in the lens focal plane and behind the focus, positions are indicated in the scheme of measurements.

Xenon has negative nonlinear Kerr coefficient at the $\mathrm{KrF}$ laser wavelength which is caused by the 2-photon resonance of 248-nm radiation with the certain $6 \mathrm{p}$ atomic state [36]. For this reason UV beam exhibits self-defocusing of filaments and intensity homogenization 
while propagating in Xe. At Xe pressure of 0.1 atm the filament diameter at the end of the cell increases by a factor 3.6 while its intensity falls down by an order of magnitude [37].

Self-defocusing of UV filaments in Xe is accompanied by the generation of coherent infrared conical emission. IR light passes through the cell output window and is directly imaged onto the profiler by means of an $\mathrm{F}=17 \mathrm{~cm}$ lens (see Figure $1 \mathrm{~b}$ ). In the focal plane, it forms multiple rings with equal radii and a constant narrow width (see Figure $2 \mathrm{~b}$ ). $\mathrm{CE}$ from each filament is collected into the ring (see layout in Figure 2). Since the filaments are inclined to the axis in a convergent laser beam CE from a bundle of filaments is displayed as a ring multitude.

Angular diameter of the rings corresponds to the apex angle of $\mathrm{CE} \Theta \approx 8 \times 10^{-3} \mathrm{rad}$, and the ring width $\Delta \Theta / \Theta \approx 0.025$ that evidences the CE monochromaticity. This angles remained unchanged for Xe pressures in the range of 0.1-2 atm. All the rings have exactly the same angular diameter although they are produced by individual filaments with somewhat different intensities. With further increase in the camera distance from the lens the rings gradually evolve into multiple spots as can be seen in panels (c) and (d) in Figure 2. These spots look unfocused and off-axis aberatted which is due to the large filament length (compared to the focal length) and their inclination to the lens axis. The IR interference patterns observed in the near-field zone indicate phase matching within the UV filament multitude and a coherent nature of the generated IR CE [32]. The energy of IR CE is found to be below the calorimeter sensitivity, i.e., less than $1 \%$ of the UV laser pulse energy.

CE spectrum is measured at $1 \mathrm{~atm}$ xenon pressure with the use of ASP 150T spectrometer (Avesta Project Ltd.) having spectral resolution of $0.15 \mathrm{~nm}$ in the IR range. To increase the dynamic range of spectra registration, a set of various filters was used to attenuate IR light. Collected spectrum is shown in Figure 3. It reveals two spectral lines of rather different intensity at $828.00 \mathrm{~nm}$ and $823.16 \mathrm{~nm}$ which can be attributed to $2 p^{5}\left({ }^{2} P_{3 / 2}\right) 6 p[1 / 2]_{0} \rightarrow 5 p^{5}\left({ }^{2} P_{3 / 2}\right) 6 s[3 / 2]_{1}^{\mathrm{o}}$ and $2 p^{5}\left({ }^{2} P_{3 / 2}\right) 6 p[1 / 2]_{2} \rightarrow 5 p^{5}\left({ }^{2} P_{3 / 2}\right) 6 s[3 / 2]_{2}^{\mathrm{o}}$ transitions of atomic $X e$, respectively [38]. In the far-field-zone CE image (see insert in Figure 3), the main bright narrow ring corresponds to the peak of 828-nm spectral line with the FWHM width $\Delta \lambda=0.25-0.30 \mathrm{~nm}$.

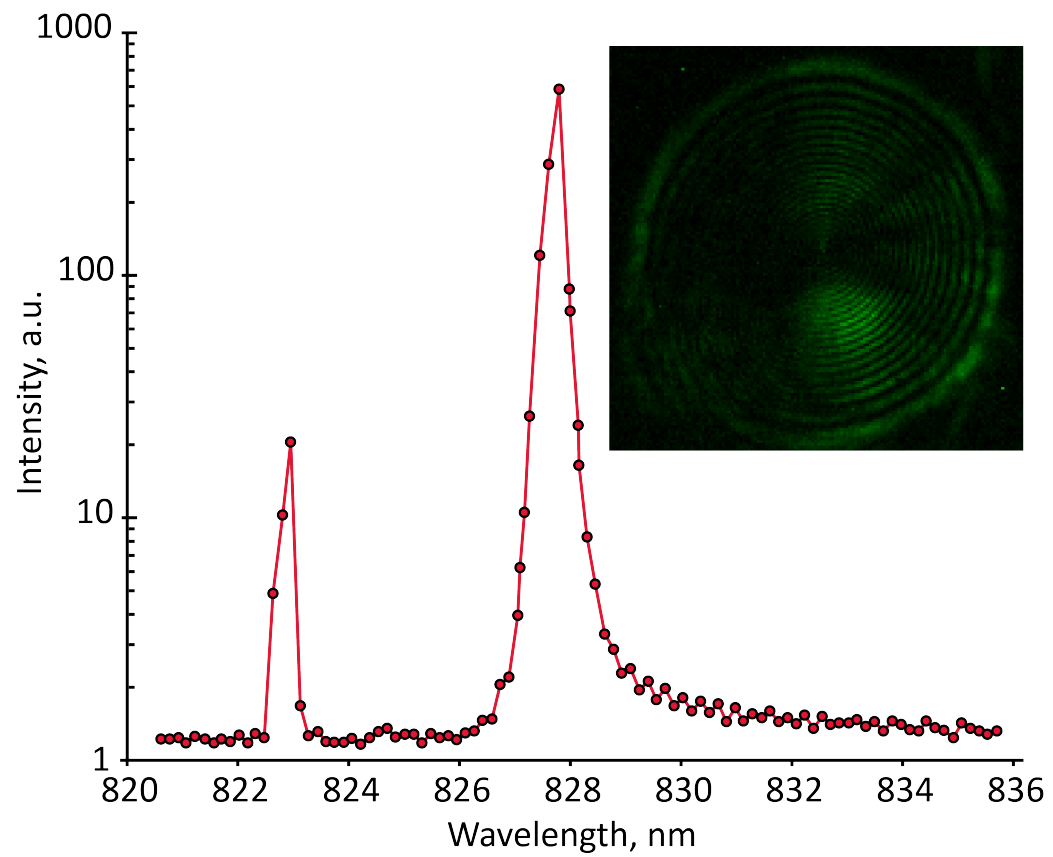

Figure 3. The IR CE spectrum in logarithmic scale and a far-field-zone CE image (in the insert). The dots are the spectrometer CCD array readings. Intensity of the inner rings (insert) was magnified by a factor of 500 . 
One can note that there exists an asymmetry of the low-intensity red-side wing of $828 \mathrm{~nm}$ spectral line. To our opinion, it results from the Xe-Xe atoms interaction and correspondent modification of the interatomic interaction potential [39,40]. Really, for the states $2 p^{5}\left({ }^{2} P_{3 / 2}\right) 6 p[1 / 2]_{0}$ and $5 p^{5}\left({ }^{2} P_{3 / 2}\right) 6 s[3 / 2]_{1}^{0}$ the significant shift of the unperturbed energy level is found to exist for $5 \AA$ interatomic distance (see Figure 9 in Ref. [41]). At atmospheric pressure the average distance between xenon atoms is only several times greater. In such situation the translational motion of atoms will give rise to modification of the line shape in the long-wave wing as well as for efficient collisional quenching of the upper state. Furthermore, one can see in Figure 3 that the long-wave wing of the spectrum is slightly modulated with $\Delta \lambda \approx 0.3 \mathrm{~nm}\left(4.5 \mathrm{~cm}^{-1}\right)$. This spectrum modulation is also displayed in the inset of Figure 3 as a series of concentric rings with three orders of magnitude less intensity. Similar spectrum modulation has been experimentally detected earlier and treated as a vibrational signature of molecular $\mathrm{Xe}_{2}^{*}$ transitions [40].

Energy states of atomic Xe involved in our interaction scheme are shown in Figure 4, namely, the ground state ${ }^{1} S_{0}(1), \mathrm{KrF}$ laser driven upper states $2 p^{5}\left({ }^{2} P_{3 / 2}\right) 6 p[1 / 2]_{0}(2)$ and $2 p^{5}\left({ }^{2} P_{3 / 2}\right) 6 p[3 / 2]_{2}\left(2^{\prime}\right)$, and intermediate states $5 p^{5}\left({ }^{2} P_{3 / 2}\right) 6 s[3 / 2]_{1}^{\mathrm{o}}(3)$ and $5 p^{5}\left({ }^{2} P_{3 / 2}\right)$ $6 s[3 / 2]_{2}^{\mathrm{O}}\left(3^{\prime}\right)$. Dipole transitions between the Xe ground (1) and excited states $(2),\left(2^{\prime}\right)$ are forbidden while two-photon transitions are allowed. Energies of the upper states (2) and (2') relative to Xe ground state 1 are $80,119 \mathrm{~cm}^{-1}$ and $79,212 \mathrm{~cm}^{-1}$, respectively, [38], which is somewhat less than a doubled energy of KrF laser photons at central wavelength $248.4 \mathrm{~nm}$ $\left(40,258 \mathrm{~cm}^{-1}\right)$. However, two-photon resonance excitation of the state (2) becomes possible owing to effective spectrum broadening in $20 \mathrm{~m}$ air pass before the gas cell. The neighbour state $\left(2^{\prime}\right)$ still remains out of the broadening interval but is populated in energy exchange collisions $(2)+(1) \rightarrow\left(2^{\prime}\right)+(1)$ due to a small energy difference of $906 \mathrm{~cm}^{-1}$ between (2) and $\left(2^{\prime}\right)$ Xe states [41]. This apparently explains 30-fold difference in intensities of $828.00 \mathrm{~nm}$ and $823.16 \mathrm{~nm}$ lines observed in our experimental conditions (see Figure 3) even though the relevant radiative transitions have comparable probabilities of $(2) \rightarrow(3)$ $3.69 \times 10^{7} \mathrm{~s}^{-1}$ and $\left(2^{\prime}\right) \rightarrow\left(3^{\prime}\right) 2.86 \times 10^{7} \mathrm{~s}^{-1}$, respectively [38]. The VUV transition from state (3) to the ground state (1) is allowed, while transition from $\left(3^{\prime}\right)$ to the ground state is forbidden. The state $\left(3^{\prime}\right)$ is depopulated in collisional exchange with the state (3) and due to formation of $\mathrm{Xe}_{2}^{*}$ dimer molecules [41].

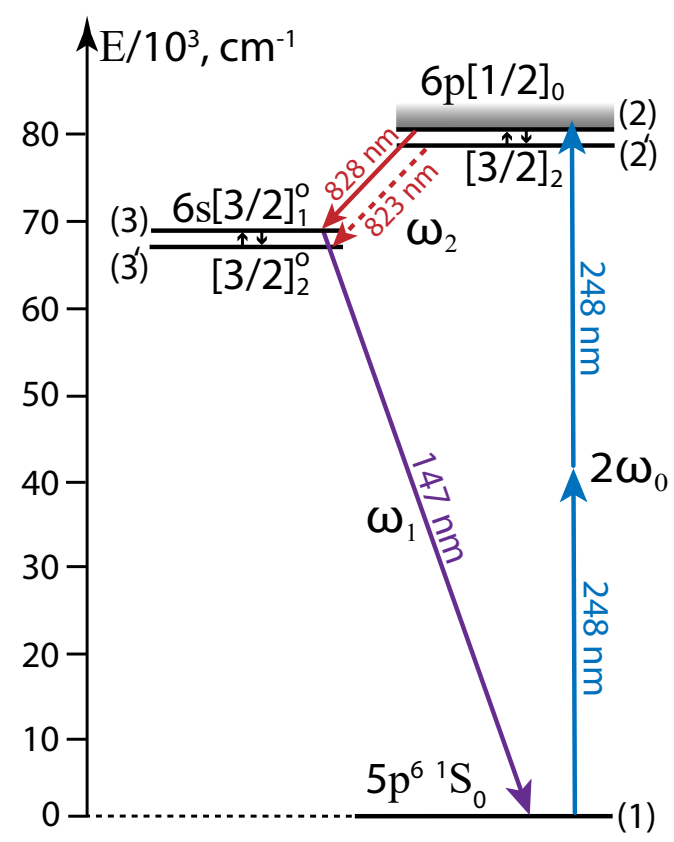

Figure 4. A diagram of atomic Xe levels involved in the coherent CE process, the energy levels are taken from [38]. A shadowed region shows a two-photon excitation band by SRRS-broadened $\mathrm{KrF}$ laser radiation. 
Below, we provide a theoretical model which describes the above mentioned experimental results. We assume that conical IR emission emerges as a result of coherent FWM process consisted in parametric conversion of two pump photons into the pair of resonance IR and VUV photons with initial duration of $\sim 1 \mathrm{ps}$, close to the pump pulse width. This coherent FWM process has a threshold in cumulative fluence of the pump $\mathrm{KrF}$ laser pulse. Due to self-induced defocusing in Xenon, the pump pulse fluence decreases below the threshold value. The FWM process is thus terminated while excitation of Xenon $2 p^{5}\left({ }^{2} P_{3 / 2}\right) 6 p[1 / 2]_{0}$ state steel proceeds through the two-photon absorption process providing population inversion of $(2) \rightarrow$ (3) transition. IR CE pulse, initially emitted as a result of coherent FWM, exhibits amplification in this inverted medium accompanied by narrowing of its spectrum up to the width determined by the reciprocal transverse relaxation time of $(2) \rightarrow(3)$ transition, $T_{2}^{-1}$. Under assumption of a spectrally-limited pulse width, the registered FWHM IR CE line width $\Delta \lambda=0.25-0.30 \mathrm{~nm}$ corresponds to temporal elongation of the amplified 828-nm CE from 1 to $\sim 10 \mathrm{ps}$.

\section{Theory of Coherent Resonance Four-Wave Mixing}

To treat the above experimental results, we will restrict ourself by the simplified three-level model taking into account only states of Xe atom that resonantly interact with the electromagnetic field (see Figure 4). Namely, the high-intensity ultrashort UV KrF laser pulse $\left(\hbar \omega_{0}=5.0 \mathrm{eV}\right)$ drives the two-photon transition between the ground state ${ }^{1} S_{0}(1)$ and the state $5 p^{5}\left({ }^{2} P_{3 / 2}\right) 6 p[1 / 2]_{0}$ (2). Stimulated IR emission emerges as a result of the dipole transition between states (2) and (3) $5 p^{5}\left({ }^{2} P_{3 / 2}\right) 6 s[3 / 2]_{1}^{\circ}\left(\hbar \omega_{2}=1.49 \mathrm{eV}, \lambda_{23}=828,0 \mathrm{~nm}\right)$ which is followed by the dipole transition into the ground state ${ }^{1} S_{0}$ accompanied with the emission of VUV quantum $\hbar \omega_{1}=8.44 \mathrm{eV}\left(\lambda_{31}=146.96 \mathrm{~nm}\right)$. As IR radiation is emitted as a hollow cone in our experiment, it indicates the crucial role of the phase synchronism condition, which allows us to consider the stimulated four-wave parametric conversion of two pump photons into the pair of resonance IR and VUV photons as the key process. As far as the pulse width of the UV KrF laser radiation is shorter than all the characteristic transverse and longitudinal relaxation time scales in the three-level system under consideration, the interaction proceeds in the coherent regime.

We will assume for definiteness that the three resonant interacting light waves in the system are of the same linear polarization. The total electric field strength is $\mathcal{E}=(1 / 2) \sum_{j=0}^{2} \mathcal{E}_{j}(\vec{r}, t) \exp \left(i \phi_{j}\right)+$ c.c.. Here, $\mathcal{E}_{j}(\vec{r}, t), j=0,1,2$, are the slow-varying complex amplitudes, $\phi_{j}=\omega_{j} t-\overrightarrow{k_{j}} \vec{r}$ are the phases, $\omega_{j}$ are the frequencies, and $\overrightarrow{k_{j}}$ are the wave vectors of the pump $\operatorname{KrF}$ laser pulse $(j=0)$, the VUV light emission $(j=1)$, and the IR cone emission $(j=2)$, respectively. Doing in conventional way, we will use the interaction Hamiltonian in the form $\hat{H}_{\text {int }}=-\hat{\mu} E$, $\hat{\mu}$ is the dipole moment operator of the Xe atom, $\mu_{12}=0$ for the dipole-forbidden transition between the atomic states (1) and (2), $\mu_{13}$ and $\mu_{23}$ are the nonzero dipole matrix elements for the transitions between the atomic states $(1,3)$ and $(2,3)$, respectively.

We seek the system wave function as the dynamic superposition of the eigen functions of atomic states,

$$
\Psi=\sum_{j=1}^{3} a_{j}(\vec{r}, t) \psi_{j}(\vec{\rho}) \exp \left(-i\left(\varepsilon_{j} / \hbar\right) t\right)+\sum_{k \geq 3} b_{k}(\vec{r}, t) \psi_{k}(\vec{\rho}) \exp \left(-i\left(\varepsilon_{k} / \hbar\right) t\right),
$$

$\varepsilon_{j}$ and $\psi_{j}(\vec{\rho})$ are the unperturbed eigen energies and functions, $\vec{\rho}$ is the intra-atomic coordinate of an electron, and $a_{j}(\vec{r}, t)$ are the slow varying amplitudes of the three resonance eigen states in this superposition, $b_{k}, k \geq 3$, are the "fast" amplitudes of none-resonance states which provide two-photon coupling between the states (1) and (2). Evolution of all these slow and fast amplitudes are guided by the above interaction Hamiltonian. As the non-resonant amplitudes $b_{k}$ oscillate at frequencies $\Omega_{k j} \pm \omega_{i}, \hbar \Omega_{k j}=\varepsilon_{k}-\varepsilon_{j}$, i.e., much faster than the field amplitudes $\mathcal{E}_{j}$ and the slow amplitudes $a_{j}$, correspondent equations can be easily time-integrated and the fast amplitudes $b_{k}$ can thus be excluded. As a result, 
we find the following system of equations for the amplitudes of states $j=1,2,3$ (the Stark shift is considered to be sufficiently small under our experimental conditions)

$$
\begin{aligned}
\frac{\partial a_{1}}{\partial t} & =i \frac{r_{12}}{2 \hbar} a_{2} \mathcal{E}_{0}^{2} e^{-i \Delta_{0}}+i \frac{\mu_{13}}{2 \hbar} a_{3} \mathcal{E}_{1} e^{-i \Delta_{1}}+i \frac{\mu_{13} \mu_{23}}{4 \hbar^{2}} a_{2} \frac{\mathcal{E}_{1} \mathcal{E}_{2} e^{-i\left(\Delta_{1}+\Delta_{2}\right)}}{\omega_{1}-\Omega_{23}} \\
\frac{\partial a_{2}}{\partial t} & =i \frac{r_{12}}{2 \hbar} a_{1} \mathcal{E}_{0}^{* 2} e^{i \Delta_{0}}+i \frac{\mu_{23}}{2 \hbar} a_{3} \mathcal{E}_{2}^{*} e^{i \Delta_{2}}+i \frac{\mu_{13} \mu_{23}}{4 \hbar^{2}} a_{1} \frac{\mathcal{E}_{1}^{*} \mathcal{E}_{2}^{*} e^{i\left(\Delta_{1}+\Delta_{2}\right)}}{\Omega_{31}-\omega_{2}} \\
\frac{\partial a_{3}}{\partial t} & =i \frac{\mu_{13}}{2 \hbar} a_{1} \mathcal{E}_{1}^{*} e^{i \Delta_{1}}+i \frac{\mu_{23}}{2 \hbar} a_{2} \mathcal{E}_{2} e^{-i \Delta_{2}}
\end{aligned}
$$

Here, the transition energies between the atomic states under consideration are $\hbar \Omega_{i j}=\varepsilon_{i}-\varepsilon_{j}$, the phase shifts are defined as follows, $\Delta_{0}=\Omega_{21} t-2 \phi_{0}, \Delta_{1}=\Omega_{31} t-\phi_{1}$, and $\Delta_{2}=\Omega_{23} t-\phi_{2}, r_{12}=\sum_{k>3}\left(\mu_{1 k} \mu_{2 k} / 2 \hbar\right)\left(\omega_{0}-\Omega_{2 k}\right)^{-1}$ is the matrix element of the two-photon transition.

To describe the dynamics of the three-level system under consideration, we need to find the elements of the effective density matrix $a_{i} a_{j}^{*}, i, j=1,2,3$. In the experiment [32], the intensity of pump $\mathrm{KrF}$ laser pulse is rather high and well exceeds intensity of the IR conical emission as well as VUV radiation. This allows us to apply the perturbation theory assuming the amplitude $\mathcal{E}_{0}$ is much larger than the amplitudes of IR and VUV light fields, $\left|\mathcal{E}_{0}\right|>>\left|\mathcal{E}_{1}\right|,\left|\mathcal{E}_{2}\right|$.

To the lowest order in this perturbation theory, system dynamics consists in coherent oscillation between the levels (1) and (2) of the two-photon transition [42]

$$
\begin{aligned}
& \left|a_{1}\right|^{2}=\cos ^{2} \tau(t), \quad\left|a_{2}\right|^{2}=\sin ^{2} \tau(t), \\
& P_{0} \equiv a_{1} a_{2}^{*} \exp \left(i \Delta_{0}\right)=-i \sin \tau \cos \tau,
\end{aligned}
$$

where the characteristic pulse area is defined as $\tau(t)=\left(r_{12} / 2 \hbar\right) \int_{-\infty}^{t} \mathcal{E}_{0}^{2}\left(t^{\prime}\right) d t^{\prime}$.

To the first order, we find no corrections to the populations $\left|a_{1}\right|^{2},\left|a_{2}\right|^{2}$ and the polarization term $P_{0}=a_{1} a_{2}^{*} \exp \left(i \Delta_{0}\right)$. The density $\left|a_{3}\right|^{2}$ remains infinitesimal, and for the polarization terms $P_{1}=a_{1} a_{3}^{*} \exp \left(i \Delta_{1}\right)$ and $P_{2}=a_{3} a_{2}^{*} \exp \left(i \Delta_{2}\right)$ we find the following set of coupled equations

$$
\begin{aligned}
& \frac{\partial P_{1}}{\partial t}-i\left(\frac{\partial \tau}{\partial t}\right) P_{2}^{*} e^{i \Delta}=-i \frac{\mu_{13}}{2 \hbar}\left|a_{1}\right|^{2} \mathcal{E}_{1}-i \frac{\mu_{23}}{2 \hbar} P_{0} \mathcal{E}_{2}^{*} e^{i \Delta} \\
& \frac{\partial P_{2}^{*}}{\partial t}-i\left(\frac{\partial \tau}{\partial t}\right) P_{1} e^{i \Delta}=-i \frac{\mu_{23}}{2 \hbar}\left|a_{2}\right|^{2} \mathcal{E}_{2}^{*}-i \frac{\mu_{13}}{2 \hbar} P_{0}^{*} \mathcal{E}_{1} e^{i \Delta} .
\end{aligned}
$$

Here, the total phase shift is $\Delta=\Delta_{0}-\Delta_{1}-\Delta_{2}$. Evidently, optimum operation corresponds to vanishing total phase shift $\Delta=0$ at each instant of time and each atom position in space. Keeping in mind the above definitions for $\Delta_{0,1,2}$, we find

$$
2 \omega_{0}=\omega_{1}+\omega_{2}, \quad 2 \vec{k}_{0}=\vec{k}_{1}+\vec{k}_{2},
$$

which are exactly the energy and momentum conservation laws for the four-wave parametric conversion process of two photons of pump radiation into the couple of scattered photons. As far as this is the resonance enhanced scattering process, the energies of scattered photons are close to the energies of allowed transitions between the atomic states (3) and (1) $\omega_{1} \approx \Omega_{31}$ and between the states (2) and (3) $\omega_{2} \approx \Omega_{23}$. Second equation in (4) results in the angle of the cone emission of the IR radiation (at the frequency $\omega_{2} \approx \Omega_{23}$ ).

Assuming natural initial conditions $P_{1,2}(t \rightarrow-\infty)=0$, we find the solutions to Equation (3) are $P_{1}=Q(t) \cos \tau(t), P_{2}=-i Q^{*}(t) \sin \tau(t)$, with the function $Q(t)$ given by

$$
Q=-i \frac{\mu_{13}}{2 \hbar} \int_{-\infty}^{t} \cos \tau\left(t^{\prime}\right) \mathcal{E}_{1}\left(t^{\prime}\right) d t^{\prime}-\frac{\mu_{23}}{2 \hbar} \int_{-\infty}^{t} \sin \tau\left(t^{\prime}\right) \mathcal{E}_{2}^{*}\left(t^{\prime}\right) d t^{\prime}
$$


Let us investigate now the process of coupled amplification of VUV and IR signals which emerges as the result of coherent FWM. Assuming the pump pulse intensity is sufficiently high, so that the pump pulse area $\tau(t)>>\left[\left(\omega_{0}-\omega_{2}\right) \tau_{p}\right]^{-1},\left[\left(\omega_{1}-\omega_{0}\right) \tau_{p}\right]^{-1}$, we find to the first order of our perturbation theory the amplitudes of medium polarization at signal frequencies $\omega_{1}$ and $\omega_{2}$ are $4 \pi \mu_{13} N P_{1}$ and $4 \pi \mu_{23} N P_{2}$, respectively, $N$ is the density of Xe atoms. We will assume below that the interaction region is not so long and one can consider the complex amplitudes $\mathcal{E}_{1,2}$ being independent on the transverse coordinates. Neglecting the group velocity dispersion, we assume both the VUV and IR signals have the same temporal shape $\Phi(\eta), \eta=t-z / v_{g}, v_{g}$ is the group velocity for both the signals. We seek solutions in the form $\mathcal{E}_{1,2}=E_{1,2}(z, \eta) \exp \left(i \vartheta_{1,2}(z, \eta)\right) \Phi(\eta)$, where the extracted real amplitudes $E_{1,2}(z, \eta)$ and phases $\vartheta_{1,2}(z, \eta)$ are assumed to be sufficiently slow varying real functions of both the variables $z$ and $\eta$. Within the slow varying amplitude and phase approximation we arrive in the following set of equations describing evolution of the amplitudes $E_{1,2}$ and the total phase $\vartheta=\vartheta_{1}+\vartheta_{2}$

$$
\begin{aligned}
& \frac{\partial E_{1}}{\partial z} \approx-\frac{2 \pi \omega_{1}^{2}}{k_{1} c^{2}} N \cos \tau\left[\frac{\mu_{13}^{2}}{2 \hbar} K_{1} E_{1}-\frac{\mu_{13} \mu_{23}}{2 \hbar} K_{2} E_{2} \sin \vartheta\right] \\
& \frac{\partial E_{2}}{\partial z} \approx \frac{2 \pi \omega_{2}^{2}}{k_{2} c^{2}} N \sin \tau\left[\frac{\mu_{23}^{2}}{2 \hbar} K_{2} E_{2}-\frac{\mu_{13} \mu_{23}}{2 \hbar} K_{1} E_{1} \sin \vartheta\right] \\
& \frac{\partial \vartheta}{\partial z} \approx 2 \pi \frac{\mu_{13} \mu_{23}}{2 \hbar c^{2}} N\left[\frac{\omega_{1}^{2}}{k_{1}} K_{2} \cos \tau \frac{E_{2}}{E_{1}}-\frac{\omega_{2}^{2}}{k_{2}} K_{1} \sin \tau \frac{E_{1}}{E_{2}}\right] \cos \vartheta .
\end{aligned}
$$

Here, we introduce two coefficients which depend on the inner coordinate $\eta, K_{1}(\eta)=$ $(1 / \Phi(\eta)) \int_{-\infty}^{\eta} \Phi\left(\eta^{\prime}\right) \cos \tau\left(\eta^{\prime}\right) d \eta^{\prime}, K_{2}(\eta)=(1 / \Phi(\eta)) \int_{-\infty}^{\eta} \Phi\left(\eta^{\prime}\right) \sin \tau\left(\eta^{\prime}\right) d \eta^{\prime}$. Note, Equation (6) are valid when the temporal shape function $\vartheta_{1,2}(z, \eta)$ do not vanishes.

According to the system (6), wave coupling in the process of coherent four-wave mixing strongly depends on correlation in the wave's phases. Evolution of the total phase $\vartheta$ is guided by the third equation in (6). It has two stationary points $\vartheta= \pm \pi / 2$, and if we assume the term in brackets is positive, the stationary point $\vartheta=\pi / 2$ turns out to be stable while the second stationary point $\vartheta=-\pi / 2$ becomes unstable. At the stationary point $\vartheta=\pi / 2$, solution to the two first equations of the system (6) under the adopted assumptions is

$$
\begin{gathered}
\frac{E_{1}}{E_{\text {seed }}}=\frac{2 \pi \omega_{1}^{2}}{k_{1} c^{2}} N \frac{\mu_{13} \mu_{23}}{2 \hbar} K_{2} \cos \tau \frac{[\exp (G z)-1]}{G} \\
\frac{E_{2}}{E_{\text {seed }}}=1+\frac{2 \pi \omega_{2}^{2}}{k_{2} c^{2}} N \frac{\mu_{23}^{2}}{2 \hbar} K_{2} \sin \tau \frac{[\exp (G z)-1]}{G}
\end{gathered}
$$

where $E_{\text {seed }}$ is the seed amplitude of the IR signal, and the exponential gain coefficient is given by

$$
G=\frac{2 \pi \omega_{2}^{2}}{k_{2} c^{2}} N \frac{\mu_{23}^{2}}{2 \hbar} K_{2} \sin \tau-\frac{2 \pi \omega_{1}^{2}}{k_{1} c^{2}} N \frac{\mu_{13}^{2}}{2 \hbar} K_{1} \cos \tau .
$$

We assume, in analogy with CE in metal vapors [7], that near threshold the signal pulses are much shorter than the pump pulse, so that $\cos \tau$ and $\sin \tau$ acquire their instant values, and $K_{1} \approx t_{s} \cos \tau, K_{2} \approx t_{s} \sin \tau, t_{s}$ is the signal pulse duration (see Figure 5). Amplification arises for that parts of the pump pulse at which the gain is positive, $G>0$, i.e.,

$$
\tan \tau>\sqrt{\frac{k_{2}}{k_{1}}} \frac{\omega_{1} \mu_{13}}{\omega_{2} \mu_{23}}
$$




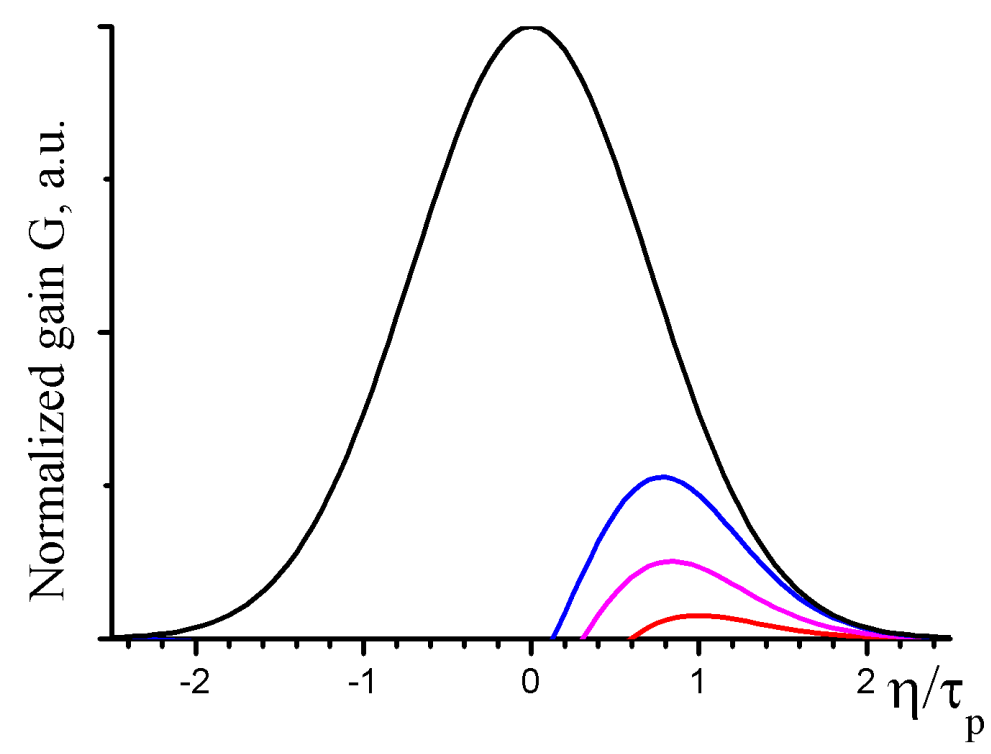

Figure 5. Normalized gain of the four-wave mixing process as a function of eigen time coordinate $\eta$ of the pump KrF laser pulse. The gain curves correspond to the pump pulse areas exceeding the threshold value by $25 \%$ (red), $50 \%$ (magenta) and $75 \%$ (blue), respectively. For comparison, the pump pulse Gaussian envelope is shown by the black line.

Let us make some estimates. The dipole matrix elements can be estimated using the data on probabilities of spontaneous emission at correspondent transitions in xenon [43] yielding $\mu_{13}=0.3576 e \cdot \AA$ and $\mu_{23}=1.87 e \cdot \AA$ with the transition wavelengths $\lambda_{13}=146.96 \mathrm{~nm}$ and $\lambda_{23}=828.0 \mathrm{~nm}$, respectively. Thus, we have the threshold pump pulse area is $\tan \left(\tau_{\text {th }}\right) \approx 0.47$. The rate of the two-photon transition between the ground state $5 p^{6}\left({ }^{1} S_{0}\right)$ and the state $5 p^{5}\left({ }^{2} P_{3 / 2}\right) 6 p[1 / 2]_{0}$ of the xenon atom has been studied in a number of experimental and theoretical works [39,40,44-47], and the results differ from each other by an order of magnitude. Estimating $r_{12} \approx \mu_{13} \mu_{23} /\left(2 \hbar\left(\omega_{0}-\Omega_{23}\right)\right.$, we find $r_{12} \approx 1.3 \times 10^{-24} \mathrm{~cm}^{3}$ while using the data [47] one finds $r_{12}=2.4 \times 10^{-24} \mathrm{~cm}^{3}$. As the above threshold pulse area is $\tau=0.47$, we find the pump laser fluence is 86 or $47 \mathrm{~mJ} / \mathrm{cm}^{2}$, respectively. At the FWHM pulsewidth of $1 \mathrm{ps}$ it corresponds to the intensity $4.6 \div 8.4 \times 10^{10} \mathrm{~W} / \mathrm{cm}^{2}$ which is well correlated with the conditions of our experiment.

\section{Spectrum Narrowing of the Amplified IR Pulse}

Pulse duration of the IR radiation generated as a result of coherent FWM process should not be larger than the KrF laser pump pulse width. However, the measured spectrum of the IR radiation corresponds to about 10 ps pulsewidth well exceeding the 1-ps KrF pump pulse duration. One can provide the following qualitative explanation to this discrepancy with the developed model.

Initially, 1-ps KrF laser pump pulse generates IR CE in coherent FWM process in high-intensity filaments and switches on phase-matching with UV filaments multitude over a large nonlinear interaction length. FWM is acocompanied by self-defocusing of filaments that reduces their intensity below the FWM threshold. However, this intensity is still high enough to provide two-photon resonance excitation of the $5 p^{5}\left({ }^{2} P_{3 / 2}\right) 6 p[1 / 2]_{0}$ state and hence to maintain the population inversion between the states (2) and (3). As a result, coherent IR CE pulse exhibits amplification while it propagates in Xe excited by low-intensity background UV radiation, and its pulsewidth thus enlarges. 
After the end of the pump pulse, interaction of the amplified IR signal with excited xenon atoms is guided by the following equations

$$
\begin{aligned}
& \frac{\partial}{\partial t}\left(\left|a_{2}\right|^{2}-\left|a_{3}\right|^{2}\right)=\frac{2 \mu_{23}}{\hbar} \operatorname{Im}\left(P_{32} \mathcal{E}_{2}\right), \\
& \frac{\partial P_{32}}{\partial t}=i \frac{\mu_{23}}{2 \hbar}\left(\left|a_{2}\right|^{2}-\left|a_{3}\right|^{2}\right) \mathcal{E}_{2}^{*}-\frac{P_{32}}{T_{2}},
\end{aligned}
$$

which can be easily derived from Equation (1) in the absence of the pump $\mathcal{E}_{0}$ and VUV $\mathcal{E}_{1}$ fields. Furthermore, the characteristic transverse relaxation time $T_{2}$ for the polarization of the resonant transition between the states (2) and (3) is phenomenologically introduced. Note, the VUV radiation does not participate in this process, moreover it exhibits strong absorption in Xe. According to our consideration in the above section, the residual population inversion of the resonant transition is

$$
\left|a_{2}\right|^{2}-\left|a_{3}\right|^{2} \approx \sin ^{2} \tau_{0}
$$

where $\tau_{0}(z)=\left(r_{12} / 2 \hbar\right) \int_{-\infty}^{\infty} \mathcal{E}_{0}^{2}\left(z, t^{\prime}\right) d t^{\prime}$ is the total pump pulse area. Note, due to selfinduced defocusing of the $\mathrm{KrF}$ laser pump pulse in xenon, the total pump pulse area $\tau_{0}(z)$ gradually decreases with the travelling distance $z$. Let the residual polarization of the transition between the states (2) and (3) be $P_{23}^{0}$, which along with Equation (11) can be considered as the initial conditions to Equation (10).

According to the results of our experiment, the IR signal amplitude remains sufficiently small throughout all the amplification process, so the population inversion does not changes significantly as a result of interaction with the IR signal. To the first order in the IR signal amplitude we find the polarization is

$$
P_{23}(t)=P_{23}^{0}-i \frac{\mu_{23}}{2 \hbar} \sin ^{2}\left(\tau_{0}\right) \exp \left(-t / T_{2}\right) \int_{-\infty}^{t} \mathcal{E}_{2}^{*}\left(t^{\prime}\right) \exp \left(t^{\prime} / T_{2}\right) d t^{\prime}
$$

Note, variation in the population inversion is of the second order in the IR signal amplitude.

Evolution of the IR signal is guided by the wave equation with the polarization term $4 \pi \mu_{23} N P_{23}$, which under the slow varying amplitude and phase approximation is

$$
\frac{\partial}{\partial z} \mathcal{E}_{2}(z, \eta) \approx i \frac{2 \pi \omega_{2}^{2}}{k_{2} c^{2}} N P_{23}(\eta),
$$

$\eta$ is the eigen time coordinate of the IR signal. Using the Fourier transform for the signal amplitude,

$$
\begin{aligned}
& \mathcal{E}_{2}(z, t)=\frac{1}{2 \pi} \int_{-\infty}^{\infty} \mathcal{E}_{2}(z, \Omega) \exp (-i \Omega t) d \Omega \\
& \mathcal{E}_{2}(z, \Omega)=\int_{-\infty}^{\infty} \mathcal{E}_{2}(z, t) \exp (-i \Omega t) d t
\end{aligned}
$$

we find the wave equation is transformed as

$$
\frac{\partial}{\partial z} \mathcal{E}_{2}(z, \Omega)=\frac{i \sin ^{2}\left(\tau_{0}(z)\right)}{\Omega T_{2}+i} \frac{E_{2}(\Omega, z)}{L_{a m p}},
$$

where the characteristic amplification length is $L_{a m p}^{-1}=\left(\pi \omega_{2}^{2} \mu_{23}^{2} N T_{2} / \hbar k_{2} c^{2}\right)$. Finally, evolution of the spectrum $S(z, \Omega)=\left|\mathcal{E}_{2}(z, \Omega)\right|^{2}$ of the amplified IR pulse is guided by the following equation

$$
S(z, \Omega)=S_{0}(\Omega) \exp \left(\frac{2}{\Omega^{2} T_{2}^{2}+1} \int_{z_{0}}^{z} \sin ^{2}\left(\tau_{0}(z)\right) \frac{d z}{L_{a m p}}\right)
$$


until it reaches the saturation. Saturation intensity $I_{\text {sat }}$ can be estimated from the condition $\mu_{23} \mathcal{E}_{2} T_{2} / \hbar=\pi$ which yields $I_{\text {sat }}=\pi \hbar^{2} \mathrm{c} /\left(8 \mu_{23}^{2} T_{2}^{2}\right) \approx 1.4 \times 10^{5} \mathrm{~W} / \mathrm{cm}^{2}$.

According to Equation (16), spectrum of the IR pulse amplitude exhibits narrowing with the distance travelled in xenon until it reaches limiting value determined by the reciprocal characteristic transverse relaxation time $T_{2}$. This process is illustrated in Figure 6 . It is assumed that initial spectrum is Gaussian with the width corresponding to the pulse duration of $1 \mathrm{ps}$, the transverse relaxation time is $T_{2}=10 \mathrm{ps}$. The pump pulse area is assumed to be constant over the amplification length $L_{a m p}$ and equals to the above threshold value, $\tan \tau=0.47$. Note, measuring of the limiting spectrum width of the amplified pulse can be considered as an independent method for experimental determination of the transverse relaxation time for a given transition: so, as a result of our experiment [32], one can conclude that the transverse relaxation time of $5 p^{5}\left({ }^{2} P_{3 / 2}\right) 6 p[1 / 2]_{0} \rightarrow 5 p^{5}\left({ }^{2} P_{3 / 2}\right) 6 s[3 / 2]_{1}^{o}$ transition in xenon at 1 atm pressure is determined to be $10 \mathrm{ps}$.

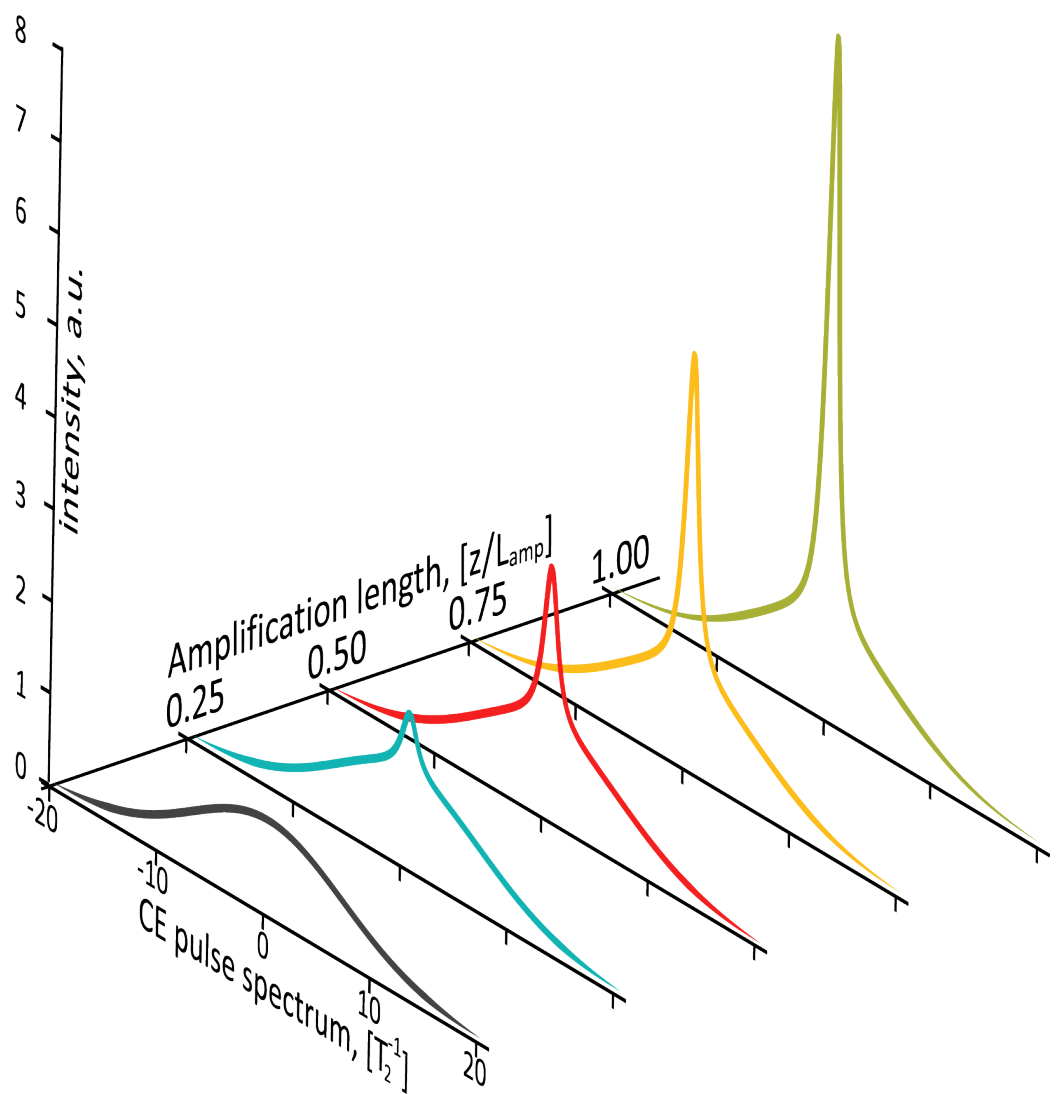

Figure 6. Spectrum narrowing of the IR cone emission pulse when it propagates in Xe excited by the under-threshold pump KrF laser pulse. Initial IR pulse spectrum is Gaussian with the width 1 ps, $T_{2}=10 \mathrm{ps}$, the pump pulse area is constant and equals to the threshold value, $\tau=0.47$.

\section{Conclusions}

We developed a theory of coherent resonance-enhanced FWM parametric difference frequency conversion of the ultrashort UV laser pulse in xenon emerging when the pulse width is less than the transverse relaxation times. It is considered the parametric frequency conversion process which proceeds through the two-photon resonance excitation of the $5 p^{5}\left({ }^{2} P_{3 / 2}\right) 6 p[1 / 2]_{0}$ state followed by stimulated coupled emission of IR $(828 \mathrm{~nm})$ and VUV $(147 \mathrm{~nm})$ quanta in $5 p^{5}\left({ }^{2} P_{3 / 2}\right) 6 p[1 / 2]_{0} \rightarrow 5 p^{5}\left({ }^{2} P_{3 / 2}\right) 6 s[3 / 2]_{1}^{\mathrm{o}}$ and $5 p^{5}\left({ }^{2} P_{3 / 2}\right) 6 s[3 / 2]_{1}^{\mathrm{o}} \rightarrow{ }^{1}$ $S_{0}$ transitions. The developed theory describes physical mechanism of narrowly directed monochromatic CE at the 828-nm wavelength produced in xenon by the filaments of $\mathrm{KrF}$ laser radiation observed earlier [32]. Initially, 1-ps KrF laser pump pulse generates IR CE in coherent FWM process in high-intensity filaments and switches on phase-matching with UV filament multitude over a large nonlinear interaction length. Self-defocusing 
of filaments reduces their intensity below the FWM threshold but this intensity is still high enough to provide two-photon resonance excitation of the $5 p^{5}\left({ }^{2} P_{3 / 2}\right) 6 p[1 / 2]_{0}$ state and hence the population inversion between the states (2) and (3). As a result, coherent IR CE pulse exhibit amplification accompanied with spectrum narrowing limited by the reciprocal transverse relaxation time $T_{2}^{-1}$.

Author Contributions: Conceptualization, I.V.S., V.D.Z. and A.M.P.; methodology, I.V.S.; formal analysis, I.V.S., A.V.B. and A.M.P.; investigation, A.V.S., N.N.U. and V.D.Z.; All authors participate in writing - original draft preparation; writing—review and editing, I.V.S., V.D.Z. and A.M.P.; supervision and project administration, I.V.S. All authors have read and agreed to the published version of the manuscript.

Funding: This research was funded by by Russian Foundation for Basic Research under the grants Nos. 18-02-00730 and 19-02-00875.

Institutional Review Board Statement: Not applicable.

Informed Consent Statement: Not applicable.

Data Availability Statement: Not applicable.

Conflicts of Interest: The authors declare no conflict of interest.

\section{Abbreviations}

The following abbreviations are used in this manuscript:

$\begin{array}{ll}\text { CE } & \text { Conical emission } \\ \text { IR } & \text { Infra-red } \\ \text { VUV } & \text { Vacuum ultra-violet } \\ \text { FWM } & \text { Four-wave mixing } \\ \text { FWHM } & \text { Full width half maximum } \\ \text { SRRS } & \text { Stimulater rotational Raman scattering }\end{array}$

\section{References}

1. Alfano, R.R.; Shapiro, S.L. Emission in the region 4000 to $7000 \AA$ A via four-photon coupling in glass. Phys. Rev. Lett. 1970, $24,584$. [CrossRef]

2. Grishkowsky, D. Self-Focusing of Light by Potassium Vapor. Phys. Rev. Lett. 1970, 24, 866. [CrossRef]

3. Harter, D.J.; Narum, P.; Raymer, M.G.; Boyd, R.W. Four-Wave Parametric Amplification of Rabi Sidebands in Sodium. Phys. Rev. Lett. 1981, 46, 1192-1195. [CrossRef]

4. Harter, D.J.; Boyd, R.W. Four-wave mixing resonantly enhanced by ac-Stark-split levels in self-trapped filaments of light. Phys. Rev. A 1984, 29, 739-748. [CrossRef]

5. Filippo, G.D.; Guldberg-Kraier, S.; Milosěvić, S.; Pedersen, J. Population of metastable barium associated with conical emission. Opt. Commun. 1997, 144, 315-321. [CrossRef]

6. Dreischuh, A.; Kamenov, V.; Dinev, S.; Reiter-Domiaty, U.; Gruber, D.; Windholz, L. Spectral and spatial evolution of a conical emission in Na vapor. J. Opt. Soc. Am. B 1998, 15, 34-40. [CrossRef]

7. Gawlik, W.; Shuker, R.; Gallagher, A. Temporal character of pulsed-laser cone emission. Phys. Rev. A 2001, 64, 021801. [CrossRef]

8. Golub, I.; Erez, G.; Shuker, R. Cherenkov emission due to laser-induced moving polarisation in sodium. J. Phys. B At. Mol. Phys. 1986, 19, L115-L120. [CrossRef]

9. Skinner, C.H.; Kleiber, P.D. Observation of anomalous conical emission from laser-excited barium vapor. Phys. Rev. A 1980, 21, 151-156. [CrossRef]

10. Ter-Mikaelian, M.; Torossian, G.; Grigoryan, G. Conical emission in the quasiresonant media as a result of self-phase modulation. Opt. Commun. 1995, 119, 56-60. [CrossRef]

11. Valley, J.F.; Khitrova, G.; Gibbs, H.M.; Grantham, J.W.; Jiajin, X. cw conical emission: First comparison and agreement between theory and experiment. Phys. Rev. Lett. 1990, 64, 2362-2365. [CrossRef]

12. Aumiler, D.; Ban, T.; Pichler, G. Femtosecond laser-induced cone emission in dense cesium vapor. Phys. Rev. A 2005, 71, 063803. [CrossRef]

13. Skenderović, H.; Ban, T.; Vujičić, N.; Aumiler, D.; Vdović, S.; Pichler, G. Cone emission induced by femtosecond excitation in rubidium vapor. Phys. Rev. A 2008, 77, 063816. [CrossRef]

14. Krasinski, J.; Gauthier, D.J.; Malcuit, M.S.; Boyd, R.W. Two-photon conical emission. Opt. Commun. 1985, 54, 241-245. [CrossRef]

15. You, L.; Mostowski, J.; Cooper, J. Cone emission from laser-pumped two-level atoms. II. Analytical model studies. Phys. Rev. A 1992, 46, 2925-2938. [CrossRef] [PubMed] 
16. Boyd, R.W.; Raymer, M.G.; Narum, P.; Harter, D.J. Four-wave parametric interactions in a strongly driven two-level system. Phys. Rev. A 1981, 24, 411-423. [CrossRef]

17. You, L.; Mostowski, J.; Cooper, J.; Shuker, R. Cone emission from laser-pumped two-level atoms. Phys. Rev. A 1991, 44, R6998R7001. [CrossRef]

18. Ben-Aryeh, Y. Cooperative effects in cone emission from laser-pumped two-level atoms. Phys. Rev. A 1997, 56, 854-858. [CrossRef]

19. Malcuit, M.S.; Gauthier, D.J.; Boyd, R.W. Suppression of Amplified Spontaneous Emission by the Four-Wave Mixing Process. Phys. Rev. Lett. 1985, 55, 1086-1089. [CrossRef]

20. Malcuit, M.S.; Gauthier, D.J.; Boyd, R.W. Competition between four-wave mixing and amplified spontaneous emission. Hyperfine Interact. 1987, 37, 125-139. [CrossRef]

21. Boyd, R.W.; Malcuit, M.S.; Gauthier, D.J.; Rzazewski, K. Competition between amplified spontaneous emission and the four-wave-mixing process. Phys. Rev. A 1987, 35, 1648-1658. [CrossRef] [PubMed]

22. Wunderlich, R.K.; Garrett, W.R.; Hart, R.C.; Moore, M.A.; Payne, M.G. Nonlinear optical processes near the sodium 4D two-photon resonance. Phys. Rev. A 1990, 41, 6345-6360. [CrossRef]

23. Garrett, W.; Hart, R.; Miller, J.; Payne, M.; Wray, J. Large spectral shifts of stimulated hyper-Raman scattering: Predictions and observations in Xe. Opt. Commun. 1991, 86, 205-210. [CrossRef]

24. Garrett, W.R.; Moore, M.A.; Hart, R.C.; Payne, M.G.; Wunderlich, R. Suppression effects in stimulated hyper-Raman emissions and parametric four-wave mixing in sodium vapor. Phys. Rev. A 1992, 45, 6687-6709. [CrossRef]

25. Garrett, W.R. Forward gain suppression of optically pumped stimulated emissions due to self-induced wave-mixing interference during a pump pulse. Phys. Rev. Lett. 1993, 70, 4059-4062. [CrossRef] [PubMed]

26. Payne, M.G.; Zhang, J.Y.; Garrett, W.R. Effect of pressure-dependent quantum interference on the ac Stark shifting of two-photon resonances. Phys. Rev. A 1993, 48, 2334-2341. [CrossRef]

27. Couairon, A.; Mysyrowicz, A. Femtosecond filamentation in transparent media. Phys. Rep. 2007, 441, 47-189. [CrossRef]

28. Bergé, L.; Skupin, S.; Nuter, R.; Kasparian, J.; Wolf, J.P. Ultrashort filaments of light in weakly ionized, optically transparent media. Rep. Prog. Phys. 2007, 70, 1633-1713. [CrossRef]

29. Béjot, P.; Kasparian, J. Conical emission from laser filaments and higher-order Kerr effect in air. Opt. Lett. 2011, 36, 4812-4814. [CrossRef] [PubMed]

30. Tünnermann, A.; Mossavi, K.; Wellegehausen, B. Nonlinear-optical processes in the near-resonant two-photon excitation of xenon by femtosecond KrF-excimer-laser pulses. Phys. Rev. A 1992, 46, 2707-2717. [CrossRef]

31. Tunnermann, A.; Momma, C.; Mossavi, K.; Windolph, C.; Welleghausen, B. Generation of tunable short pulse VUV radiation by four-wave mixing in xenon with femtosecond KrF-excimer laser pulses. IEEE J. Quantum Electron. 1993, 29, 1233-1238. [CrossRef]

32. Zvorykin, V.D.; Goncharov, S.A.; Ionin, A.A.; Mokrousova, D.V.; Ryabchuk, S.V.; Seleznev, L.V.; Shutov, A.V.; Smetanin, I.V.; Sunchugasheva, E.S.; Ustinovskii, N.N. Kerr self-defocusing of multiple filaments in TW peak power UV laser beam. Laser Phys. Lett. 2016, 13, 125404. [CrossRef]

33. Zvorykin, V.D.; Levchenko, A.O.; Ustinovskii, N.N. Amplification of subpico second UV pulses in the multistage GARPUN-MTW Ti:sapphire-KrF laser system. Quantum Electron. 2010, 40, 381-385. [CrossRef]

34. Zvorykin, V.; Ionin, A.; Mokrousova, D.; Seleznev, L.; Smetanin, I.; Sunchugasheva, E.; Ustinovskii, N.; Shutov, A. Range of multiple filamentation of a terawatt-power large-aperture KrF laser beam in atmospheric air. J. Opt. Soc. Am. B 2019, 36, G25-G32. [CrossRef]

35. Smetanin, I.; Levchenko, A.; Shutov, A.; Ustinovskii, N.; Zvorykin, V. Role of coherent resonant nonlinear processes in the ultrashort $\mathrm{KrF}$ laser pulse propagation and filamentation in air. Nucl. Instruments Methods Phys. Res. Sect. B Beam Interact. Mater. Atoms 2016, 369, 87-91. [CrossRef]

36. Lehmberg, R.; Pawley, C.; Deniz, A.; Klapisch, M.; Leng, Y. Two-photon resonantly-enhanced negative nonlinear refractive index in xenon at $248 \mathrm{~nm}$. Opt. Commun. 1995, 121, 78-88. [CrossRef]

37. Zvorykin, V.D.; Shutov, A.V.; Ustinovskii, N.N. Review of nonlinear effects under TW-power PS pulses amplification in GARPUN-MTW Ti:sapphire-KrF laser facility. Matter Radiat. Extrem. 2020, 5, 045401. [CrossRef]

38. Kramida, A.; Ralchenko, Y.; Reader, J.; NIST ASD Team. NIST Atomic Spectra Database (ver. 5.7.1). Available online: https:/ / physics.nist.gov/asd (accessed on 27 April 2020). [CrossRef]

39. Raymond, T.D.; Böwering, N.; Kuo, C.Y.; Keto, J.W. Two-photon laser spectroscopy of xenon collision pairs. Phys. Rev. A 1984, 29, 721-734. [CrossRef]

40. Gornik, W.; Kindt, S.; Matthias, E.; Schmidt, D. Two-photon excitation of xenon atoms and dimers in the energy region of the $5 p^{5} 6 p$ configuration. J. Chem. Phys. 1981, 75, 68-74. [CrossRef]

41. Böwering, N.; Bruce, M.R.; Keto, J.W. Collisional deactivation of two-photon laser excited xenon $5 p^{5} 6 p$. II. Lifetimes and total quench rates. J. Chem. Phys. 1986, 84, 715-726. [CrossRef]

42. Belenov, É.M.; Kryukov, P.G.; Nazarkin, A.V.; Smetanin, I.V. Photoionization of a gas by an ultrashort laser pulse with two-photon excitation of an intermediate level. Sov. J. Quantum Electron. 1992, 22, 1113-1116. [CrossRef]

43. Aymar, M.; Coulombe, M. Theoretical transition probabilities and lifetimes in Kr I and Xe I spectra. At. Data Nucl. Data Tables 1978, 21, 537-566. [CrossRef]

44. Chen, C.; Hurst, G.; Payne, M. Direct counting of Xe atoms. Chem. Phys. Lett. 1980, 75, 473-477. [CrossRef] 
45. Hawkins, R.T.; Egger, H.; Bokor, J.; Rhodes, C.K. A tunable, ultrahigh spectral brightness KrF* excimer laser source. Appl. Phys. Lett. 1980, 36, 391-392. [CrossRef]

46. Kröll, S.; Bischel, W.K. Two-photon absorption and photoionization cross-section measurements in the $5 p^{5} 6 \mathrm{p}$ configuration of xenon. Phys. Rev. A 1990, 41, 1340-1349. [CrossRef]

47. Pindzola, M.S.; Payne, M.G.; Garrett, W.R. Two-photon excitation of xenon. Phys. Rev. A 1981, 24, 3115-3119. [CrossRef] 